\title{
Robustness of Nonlinear Control Systems to Network-Induced Imperfections
}

\author{
Domagoj TOLIĆ, Ivana PALUNKO
}

\begin{abstract}
Nowadays control systems are increasingly implemented over shared resource-constrained communication networks. Namely, sensors, controllers and actuators no longer exchange information through dedicated point-to-point connections but compete for network access, which gives rise to network-induced imperfections that adversely affect control performance. Prevalent network phenomena are scheduling protocols, nonuniform variable delays, quantization, packet dropouts, sampled and distorted data. Besides possessing usual robustness requirements (e.g., to modeling uncertainties or external disturbances), such control systems ought to be robust against the aforementioned network phenomena as well. This article brings a methodology to quantify control system robustness via $L_{p}$-gains as the control laws, communication delays, sampling intervals, noise levels or scheduling protocols change. Building upon impulsive delayed system modeling, Lyapunov stability and the small-gain theorem, the proposed methodology takes into account nonlinear time-varying dynamic controllers and plants as well as model-based estimation, output feedback and large delays. The inverted pendulum example is provided.
\end{abstract}

Keywords: impulsive delayed system; $L_{p}$-stability; networked control systems; robust control

\section{INTRODUCTION}

With the advent of more advanced hardware and software components, modern control systems are frequently being implemented over a shared (wired or wireless) resource-constrained communication network [1-9]. In particular, spatially distributed sensors, controllers and actuators in such control systems, termed Networked Control Systems (NCSs), do not exchange information through dedicated point-to-point connections. Instead, owing to the limited capacity of the network, control system components need to compete for network access [4, 10]. On the one hand, NCSs offer greater flexibility as well as reduced implementation and maintenance costs in comparison with traditional control systems. On the other hand, network-induced imperfections present in NCSs lead to impaired control system performance and may even lead to instability. Commonly investigated network-induced phenomena are scheduling protocols, nonuniform time-varying delays, quantization, packet dropouts, aperiodic sampling and noisy data. Consequently, in addition to possessing the usual robustness requirements (e.g., to modeling uncertainties and/or external disturbances), NCSs also need to be robust against these network-induced imperfections. As expected, the control system community addresses these additional robustness requirements by focusing on control design and imposing more stringent demands on control laws.

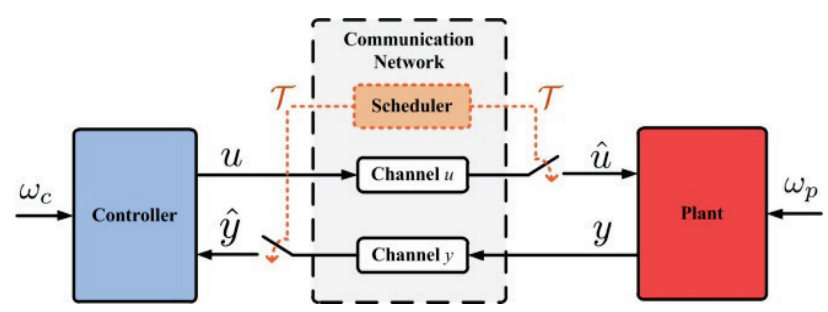

Figure $1 \mathrm{~A}$ control system consisting of the plant and controller that interact over a communication network. As indicated by the two switches, the information between the controller and plant is exchanged intermittently (as orchestrated by some scheduling protocol between the NCS links) at discrete time instants in asset $\mathcal{T}$. The NCS links are characterized by time-varying, and possibly different, delays.
The following problem is investigated in this article. Consider a time-varying nonlinear control system comprised of a controller with delayed dynamics

$\dot{x}_{c}=f_{c}\left(t, x_{c_{t}}, \hat{y}, \omega_{c}\right), u=g_{c}\left(t, x_{c_{t}}\right)$,

and of a plant with delayed dynamics

$\dot{x}_{p}=f_{p}\left(t, x_{p_{t}}, \hat{u}, \omega_{p}\right), u=g_{p}\left(t, x_{p_{t}}\right)$,

where $x_{c} \in \mathbb{R}^{n_{c}}$ and $x_{p} \in \mathbb{R}^{n_{p}}$ are the states, $u \in \mathbb{R}^{n_{u}}$ and $y \in \mathbb{R}^{n_{y}}$ are the outputs, and $\left(y, \omega_{c}\right) \in \mathbb{R}^{n_{y} \times n_{\omega_{c}}}$ and $\left(u, \omega_{p}\right) \in \mathbb{R}^{n_{u} \times n_{\omega_{p}}}$ are the inputs of the controller and plant, respectively, where $\omega_{c}$ and $\omega_{p}$ are controller and plant modeling uncertainties and/or external disturbances. The controller and plant are interconnected according to Fig. 1, that is, the quantity $\hat{y}$ is the distorted and delayed version of $y$ received by the controller (1) while the quantity $\hat{u}$ is the distorted and delayed control input $u$ fed to the plant (2). The translation operators $x_{c_{t}}$ and $x_{p_{t}}$, defined in Section 2.2, are with the corresponding controller and plant delays $d_{c} \geq 0$ and $d_{p} \geq 0$, respectively. The main goal of this work is to find/estimate the $\mathrm{L}_{p}$-gain, where $p \in[1, \infty]$, from $\left(\omega_{c}, \omega_{p}\right)$ to $\left(x_{c}, x_{p}\right)$ for the NCS (1)-(2) in the presence of some sampling intervals, model-based estimators, noise levels, scheduling protocols and NCS link delays.

This article brings a methodology to quantify control system robustness (in terms of $\mathrm{L}_{p}$-gains) for various underlying control laws, communication delays, sampling periods, noise levels or scheduling protocols. Building upon impulsive delayed system modeling, Lyapunov stability and the small-gain theorem, the proposed methodology takes into account nonlinear time-varying dynamic control laws and plants as well as Uniformly Globally Exponentially Stable (UGES) scheduling protocols, intermittent data exchange, noise, output feedback, model-based estimation and nonuniform timevarying large delays. Delays greater than the transmission intervals are called large delays. Taking the utilized robustness tool into account, other approaches pertaining to robustness of NCSs can be classified as: i) small-gain 
theorem approaches [7-9, 11-13]; ii) dissipativity and passivity-based approaches [14]; and iii) Input-to-State Stability (ISS) approaches [15-17]. Since the corresponding robustness notions are distinct properties for nonlinear (delayed) dynamic systems, each work sheds different light on the NCS robustness problem for a specific class of NCSs.

The main contributions of the present work are twofold:

a) the Lyapunov-based procedure for assessing robustness of NCSs via $\mathrm{L}_{p}$-gains with respect to the commonly encountered network-induced imperfections; and b) the large class of control systems and network phenomena considered. In comparison with [18], we consider large delays and noisy/distorted data. Furthermore, [19] investigates time-varying nonlinear plants that are affine in control (no delayed dynamics in the controller nor plant) with constant delays and state feedback. In addition, [19] does not take into account modeling/exogenous disturbances, distorted data or scheduling protocols. The authors in [20] and [21] focus on linear control systems and Zero-Order-Hold ( $\mathrm{ZOH})$ sampling (rather than model-based estimation) whilst neither noisy data nor scheduling protocols are considered. It is also worth mentioning that [20] does not take into account disturbances. Similar observations can be made with respect to other related works (see [6], [11], [14-19, 22, 23] and the references therein). In comparison with [9], the present work focuses entirely on NCS robustness even though similar mathematical tools are utilized. The conference version of this article is [24].

The remainder of the article is organized as follows. Section 2 presents the notation and mathematical preliminaries employed herein. A detailed exposition of the network-induced phenomena modeling is found in Section 3. Section 4 devises methodology for measuring robustness in terms of $\mathrm{L}_{p}$-gains for a class of NCSs. The inverted pendulum stabilization problem is provided in Section 5 to demonstrate the effectiveness of our methodology. Conclusions and future research avenues are in Section 6.

\section{PRELIMINARIES}

\subsection{Notation}

The dimension of a vector $x$ is denoted $n_{x}$, while $\|x\|$ denotes its Euclidean norm. The $n$-dimensional null vector is $\mathbf{0}_{n}$, while $\mathbb{R}_{+}^{n}$ denotes the nonnegative orthant. The concatenation of vectors $x \in \mathbb{R}^{n_{x}}$ and $y \in \mathbb{R}^{n_{y}}$ is $(x, y)$. Let

$\|f[a, b]\|_{p}:=\left(\int_{a}^{b}\|f(s)\|^{p} \mathrm{~d} s\right)^{\frac{1}{p}}$,

where $p \in[1, \infty]$, denote the $\mathrm{L}_{p}$-norm of a Lebesgue measurable function $f: \mathbb{R} \rightarrow \mathbb{R}^{n}$ on the interval $[a, b] \subset$ $\mathbb{R}$. If the previous expression is finite, we write $f \in$ $L_{p}[a, b]$. Likewise, we write $f \in L_{p}$ when the above norm is finite for any $a, b \in \mathbb{R}$.

Left-hand and right-hand limits are denoted $x\left(t^{-}\right)=$ $\lim _{t^{\prime} \lambda t} x\left(t^{\prime}\right)$ and $x\left(t^{+}\right)=\lim _{t^{\prime} \backslash t} x\left(t^{\prime}\right)$, respectively. Next, for a set $S \subseteq \mathbb{R}^{n}$,let $P C([a, b], S)=\{\phi:[a, b] \longrightarrow$
$S \mid \phi(t)=\phi\left(t^{+}\right)$for every $t \in[a, b), \phi\left(t^{-}\right)$exists in $S$ for all $t \in(a, b]$ and $\phi\left(t^{-}\right)=\phi(\mathrm{t})$ for all but at most a finite number of points $t \in(a, b]\}$.

\subsection{Delayed Systems with Impulses}

We investigate a nonlinear impulsive delayed system

$\Sigma\left\{\begin{array}{r}\chi\left(t^{+}\right)=h_{\chi}\left(t, \chi_{t}\right) \quad t \in \mathcal{T} \\ \dot{\chi}(t)=f_{\chi}\left(t, \chi_{t}, \omega\right) \\ y=l_{\chi}\left(t, \chi_{t}, \omega\right)\end{array}\right\}$ otherwise,

where $\omega \in \mathbb{R}^{n_{\omega}}$ is the input, $y \in \mathbb{R}^{n_{y}}$ is the output, and $\chi \in \mathbb{R}^{n_{\chi}}$ is the state of the system. We consider regular enough functions $f_{\chi}$ and $h_{\chi}$ to guarantee forward completeness of solutions which, given initial condition $\chi_{t_{0}} \in P C\left([-d, 0], \mathbb{R}^{n_{\chi}}\right)$ at $t_{0}$, where $d \geq 0$ is the maximum value of all time-varying delay phenomena, are given by right-continuous functions $t \rightarrow \chi(t) \in$ $P C\left(\left[t_{0}-d, \infty\right], \mathbb{R}^{n_{\chi}}\right)$. Also, $\chi_{t}$ is the translation operator acting on the trajectory $\chi(\cdot)$ and is defined by $\chi_{t}(\theta)$ $:=\chi(t+\theta)$ for $-d \leq \theta \leq 0$. In other words, $\chi_{t}$ is the restriction of $\chi(\cdot)$ to the interval $[t-d, t]$ translated to $[-d, 0]$. The norm of $\chi_{t} \in P C\left([-d, 0], \mathbb{R}^{n_{\chi}}\right)$ is $\left\|\chi_{t}\right\|=\sup _{-d \leq \theta \leq 0}\left\|\chi_{t}(\theta)\right\|$. Jumps/impulses of the state, denoted $\chi\left(t^{+}\right)$, take place at time instants $t \in \mathcal{T}$ $:=\left\{t_{1}, t_{2}, \ldots\right\}$, where $\varepsilon \leq t_{i+1}-i_{i} \leq \tau$, for each $i \in \mathbb{N}_{0}$ with $\varepsilon>0$ arbitrarily small. Upon a jump, the state value becomes $\chi\left(t^{+}\right)$. The considered solutions of $\Sigma$ follow [25, Chapter $2 \& 3]$.

Definition 1 (Uniform Global Exponential Stability): For $\omega \equiv \mathbf{0}_{n_{\omega}}$, the system $\Sigma$ is said to be Uniformly Globally Exponentially Stable (UGES) if there exist positive constants $\lambda$ and $M$ such that, for each $t_{0} \in \mathbb{R}$ and each $\chi_{t_{0}} \in P C\left([-d, 0], \mathbb{R}^{n_{\chi}}\right)$, each solution $t \rightarrow \chi(t) \in$ $P C\left(\left[t_{0}-d, \infty\right], \mathbb{R}^{n_{\chi}}\right)$ to $\Sigma$ satisfies $\|\chi(t)\| \leq$ $M\left\|\chi_{t_{0}}\right\| e^{-\lambda\left(t-t_{0}\right)}$ for each $t \geq t_{0}$.

Definition $2\left(\mathrm{~L}_{p}\right.$-Stability with Biasb): Let $p \in[1, \infty]$. The system $\Sigma$ is $\mathrm{L}_{p}$-stable with bias $b(t) \equiv b \geq 0$ from $\omega$ to $y$ with $\mathrm{L}_{p}$-gain $\gamma \geq 0$ if there exists $K \geq 0$ such that, for each $t_{0} \in \mathbb{R}$ and each $\chi_{t_{0}} \in P C\left([-d, 0], \mathbb{R}^{n_{\chi}}\right)$, each solution to $\Sigma$ from $\chi_{t_{0}}$ satisfies $\left\|y\left[t_{0}, t\right]\right\|_{p} \leq \mathrm{K}\left\|\chi_{t_{0}}\right\|+$ $\gamma\left\|\omega\left[t_{0}, t\right]\right\|_{p}+\left\|b\left[t_{0}, t\right]\right\|_{p}$ for each $t \geq t_{0}$.

Definition $3\left(\mathrm{~L}_{p}\right.$-Detectability): Let $p \in[1, \infty]$. The state $\chi$ of $\Sigma$ is $\mathrm{L}_{p}$-detectable from $(y, \omega)$ with gain $\gamma \geq 0$ if there exists $K \geq 0$ such that, for each $t_{0} \in \mathbb{R}$ and each $\chi_{t_{0}} \in P C\left([-d, 0], \mathbb{R}^{n} \chi\right)$, each solution to $\Sigma$ from $\chi_{t_{0}}$ satisfies $\quad\left\|\chi\left[t_{0}, t\right]\right\|_{p} \leq \mathrm{K}\left\|\chi_{t_{0}}\right\|+\gamma\left\|\omega\left[t_{0}, t\right]\right\|_{p}+$ $\gamma\left\|y\left[t_{0}, t\right]\right\|_{p}$ for each $t \geq t_{0}$.

A nonzero bias $b$ is caused by noisy and corrupted data [7, 9]. Hence, when the data is not noisy or corrupted, we say " $\mathrm{L}_{p}$-stability" rather than " $\mathrm{L}_{p}$-stability with bias 0 ". Definitions 2 and 3 are motivated by $[11,26]$.

\section{MODELLING NETWORK-INDUCED IMPERFECTIONS}

In this section, we provide a step-by-step modeling of network-induced imperfections. To that end, we partition the vector $(y, u)$ such that each partition block corresponds to one NCS link. In other words, all 
components of $(y, u)$ within the same partition block are sampled at same time instants and experience the same delays. Apparently, if we take lto denote the number of partition blocks, one can infer that $l \in\left\{1, \ldots, n_{y}+n_{u}\right\}$. Consequently, the partition blocks of $(y, u)$ are indexed with the set $\{1, \ldots, l\}$.

\subsection{Delayed Information}

Let us introduce the translation operators $y_{t}$ and $u_{t}$ with the maximal network-induced delay $d \geq 0$ arising, for example, from propagation and/or protocol arbitration. According to the above discussion, the operator $\left(y_{t}, u_{t}\right)$ delays each partition block of $(y, u)$ for the associated delay. Essentially, if the $i^{\text {th }}$ partition block of $(y(t), u(t))$, that is $(y(t), u(t))_{i}, \quad i \in\{1, \ldots, l\}$, is transmitted with delay $d_{i}: \mathbb{R} \rightarrow \mathbb{R}_{+}$, then the $i^{\text {th }}$ partition block of $\left(y_{t}, u_{t}\right)$, that is $\left(y_{t}, u_{t}\right)_{i}$, is in fact $\left(y\left(t-d_{i}(t)\right), u\left(t-d_{i}(t)\right)\right)_{i}$. We assume $d_{i}(t)$ 's are differentiable, with an upper bound on $\left|\dot{d}_{i}(t)\right|$, and upper bounded by $d$ so that $d:=\max \left\{\sup _{t \in \mathbb{R}} d_{1}(t), \ldots, \sup _{t \in \mathbb{R}} d_{l}(t)\right\}$. We are now ready to define the following error vector

$e=\left[\begin{array}{l}e_{y}(t) \\ e_{u}(t)\end{array}\right]:=\left[\begin{array}{l}\hat{y}(t)-y_{t} \\ \hat{u}(t)-u_{t}\end{array}\right]$

\subsection{Intermittent and Noisy Information Updates}

When the most recent data, corresponding to partition blocks of $(y, u)$, arrives at time instants $t_{1}, t_{2}, \ldots, t_{i}, \ldots \in$ $\mathcal{T}$, the respective components of $(\hat{y}, \hat{u})$ are updated, i.e.,

$\left.\begin{array}{l}\hat{y}\left(t_{i}^{+}\right)=y_{t}+h_{y}\left(t_{i}, e\left(t_{i}\right)\right) \\ \hat{u}\left(t_{i}^{+}\right)=u_{t}+h_{u}\left(t_{i}, e\left(t_{i}\right)\right)\end{array}\right\} t_{i} \in \mathcal{T}$,

where $h_{y}: \mathbb{R} \times \mathbb{R}^{n_{e}} \rightarrow \mathbb{R}^{n_{y}}$ and $h_{u}: \mathbb{R} \times \mathbb{R}^{n_{e}} \rightarrow \mathbb{R}^{n_{u}}$ capture the underlying scheduling protocol, measurement noise and channel distortion. Namely, when the $j^{\text {th }} \mathrm{NCS}$ link, $j \in\{1, \ldots, l\}$, is awarded the medium access at some time instant $t_{i} \in \mathcal{T}$, the associated partition block of $\left(\hat{y}\left(t_{i}\right), \hat{u}\left(t_{i}\right)\right)$ jumps to the received values, while all other partition blocks of $\left(\hat{y}\left(t_{i}\right), \hat{u}\left(t_{i}\right)\right)$ remain intact. Also, the associated block of $e\left(t_{i}\right)$ jumps to the noise values $v_{j}\left(t_{i}\right)$ carried by the received data, i.e.,

$e_{j}\left(t_{i}^{+}\right)=v_{j}\left(t_{i}\right)$

Moreover, we assume

$\sup _{t \in \mathbb{R}, j \in\{1, \ldots, l\}}\left\|v_{j}(t)\right\|=K_{v}$.

Noise $v_{j}\left(t_{i}\right)$, embedded in $h_{y}$ and $h_{u}$, models any discrepancy between the received values and their actual values at time $t_{i}-d_{j}\left(t_{i}\right)$ (when the $j^{\text {th }}$ partition block of $(y(t), u(t))$ was sampled). This discrepancy can be a consequence of measurement noise and channel distortion. We stress that $v_{j}$ is by no means related to $\left(\omega_{p}, \omega_{c}\right)$ and that out-of-order packet arrivals, which are possible due to time-varying delays, are allowed for.

\subsection{UGES Scheduling Protocols}

The definition of UGES scheduling protocols below is derived from [11] and [18].

Definition 4: Assume no-noise setting, that is, $K_{v}=$ 0 . The protocol captured with $h:=\left(h_{y}, h_{u}\right)$ is UGES if there exists a function $W: \mathbb{N}_{0} \times \mathbb{R}^{n_{e}} \rightarrow \mathbb{R}_{+}$with $W(i$, ): $\mathbb{R}^{n_{e}} \rightarrow \mathbb{R}^{+}$locally Lipschitz for alli $\in \mathbb{N}_{0}$, and if there exist $a, \bar{a}>0$ and $0 \leq \rho<1$ such that $\underline{a}\|e\| \leq W(i, e) \leq$ $\bar{a}\|e\|$, and $W\left(i+1, h\left(t_{i}, e\right)\right) \leq \rho W(i, e)$, for $\operatorname{each}(i, e) \in$ $\mathbb{N}_{0} \times \mathbb{R}^{n_{e}}$.

Observe that $i$ in $W(i, e)$ is the number of jumps (i.e., transmissions) and corresponds to $t_{i} \in \mathcal{T}$. Furthermore, although the delays might be a consequence of protocol arbitration, the delays do not constitute Definition 4 [11], [18]. Typical UGES protocols are the Round Robin (RR) and Try-Once-Discard protocol (TOD) as discussed in $[10,11,18,27]$. RR is an example of static scheduling, in which there exists a predefined transmission pattern that repeats cyclically, while TOD is an example of dynamic scheduling, in which the medium access is granted to the NCS link with the greatest value of $\left\|e_{i}\right\|, i \in\{1, \ldots, l\}$.

\subsection{Model-Based Estimation}

Between two consecutive transmissions, the values of $\hat{u}$ and $\hat{y}$ do not have to be kept constant. Instead, the values of $\hat{u}$ and $\hat{y}$ may be estimated to obtain greater robustness margins $[9,28]$. When model-based estimation is employed, then for each $t \in\left[t_{0}, \infty\right) \backslash \mathcal{T}$

$\dot{\hat{u}}=\hat{f}_{c}\left(t, x_{p_{t}}, x_{c_{t}}, \hat{y}_{t}, \hat{u}_{t}, \omega_{p}, \omega_{c}\right)$,

$\dot{\hat{y}}=\hat{f}_{p}\left(t, x_{p_{t}}, x_{c_{t}}, \hat{y}_{t}, \hat{u}_{t}, \omega_{p}, \omega_{c}\right)$

where the translation operators $\hat{u}_{t}$ and $\hat{y}_{t}$ are with delay $d$. The scenario without estimation corresponds to the $\mathrm{ZOH}$ strategy and is characterized by $\dot{\hat{u}} \equiv \mathbf{0}_{n_{u}}$ and $\dot{\hat{y}} \equiv \mathbf{0}_{n_{y}}$.

\section{METHODOLOGY}

The proofs of the technical results of this section are based on the proofs found in the accompanying technical report of [9]. The link to this technical report is provided in [9]. Hence, owing to the space constraints, we omit the proofs. In addition, the reader is referred to [9] for a link between the $\mathrm{L}_{p}$-stability results presented below and Uniform Global Stability (UGS) as well as Global Asymptotic Stability (GAS) of NCSs.

\subsection{Rewriting the Closed-Loop System}

Before proceeding further, let us introduce the stack vectors $x:=\left(x_{p}, x_{c}\right)$ and $\omega:=\left(\omega_{p}, \omega_{c}\right)$. Following [11], we rewrite the control system (1)-(2) as follows:

$$
\left.\begin{array}{c}
\left.\begin{array}{c}
x\left(t^{+}\right)=x(t) \\
e\left(t^{+}\right)=h(t, e(t))
\end{array}\right\} \quad t \in \mathcal{T} \\
\dot{x}=f\left(t, x_{t}, e, \omega\right) \\
\dot{e}=g\left(t, x_{t}, e_{t}, \omega_{t}\right)
\end{array}\right\} \text { otherwise, }
$$


where functions $f, g$ and $h$ are explicitly written in (6) and (7) and are supposed to possess enough regularity to guarantee existence of the solutions on the interval of interest [25, Chapter 3]. From (7), one can see why differentiability of $d_{i}(t)$ 's and boundedness of $\left|\dot{d}_{i}(t)\right|$ are needed in attaining regularity of $g$. For notational convenience, we do not explicitly distinguish among translation operators with delays $d_{p}, d_{c}, d$ or $2 d$ in (6), (7) and the remainder of this article. Nevertheless, let us point out that the operators $x_{c_{t}}$ and $x_{p_{t}}$ are with delays $d_{c}$ and $d_{p}$, respectively, the operators $g_{c_{t}}$ and $g_{p_{t}}$ in $\hat{f}_{c}$ and $\hat{f}_{p}$ are with delay $2 d$ whilst all other operators are with delay $d$. We also use $\bar{d}:=2 d+\max \left\{d_{p}, d_{c}\right\}$ to denote the maximum value of all delay phenomena in (7).

Let us group together the dynamics

$\left.e\left(t^{+}\right)=h(t, e(t))\right\} \quad t \in \mathcal{T}$

$\left.\dot{e}=g\left(t, x_{t}, e_{t}, \omega_{t}\right)\right\}$ otherwise,

and label it error system $\Sigma_{e}$. In addition, let us group together the dynamics

$\left.x\left(t^{+}\right)=x(t)\right\} \quad t \in \mathcal{T}$

$\left.\dot{x}=f\left(t, x_{t}, e, \omega\right)\right\}$ otherwise,

and label it nominal system $\Sigma_{n}$. Observe that $\Sigma_{n}$ contains delays, but does not depend on $\mathcal{T}$ nor $h$. Instead, $\mathcal{T}$ and $h$ constitute the error subsystem $\Sigma_{e}$.

The remainder of our methodology interconnects $\Sigma_{n}$ and $\Sigma_{e}$ and applies the small-gain theorem [29, Chapter 5]. As shown below, $W(i, e)$ from Definition 4 becomes the output of $\Sigma_{e}$ while the output of $\Sigma_{n}$, denoted $H\left(x_{t}, \omega_{t}\right)$, is obtained combining $g\left(t, x_{t}, e_{t}, \omega_{t}\right)$ and $W(i, e)$. The proposed system interconnection is merely a convenience, since the outputs $H\left(x_{t}, \omega_{t}\right)$ and $W(i, e)$ do not exist physically, to employ the small-gain theorem and obtain $\mathrm{L}_{p}$-gain from $\omega$ to $x$.

\section{2 $L_{p}$-Stability of Delayed LTI Systems with Impulses}

In this subsection, given some delay $d(t)$ and the upper bound $\tau$ on transmission intervals, we utilize the Lyapunov stability theory to compute/estimate the $\mathrm{L}_{p}$-gain for a class of impulsive delayed LTI systems. Afterward, we apply the results of this subsection toward computing/estimating $\mathrm{L}_{p}$-gains of the error subsystem $\Sigma_{e}$.

Consider the following delayed LTI system with impulses

$$
\begin{array}{ll}
\dot{\xi}(t)=a \xi(t-d(t))+\tilde{u}(t), & t \notin \mathcal{T}, \\
\xi\left(t^{+}\right)=c \xi(t)+\tilde{v}(t), & t \in \mathcal{T},
\end{array}
$$

where $\in(-1,1)$ and $a \in \mathbb{R}$, with initial conditions $\xi_{t_{0}} \in P C([-\breve{d}, 0], \mathbb{R})$. Next, suppose that $d(t)$ is a continuous function upper bounded by $\breve{d}$ while $\tilde{u}, \widetilde{v}: \mathbb{R} \rightarrow$ $\mathbb{R}$ are external inputs with the property $\tilde{v} \in L_{\infty}$.

Lemma 1: Suppose $\tilde{u} \equiv 0, \tilde{v} \equiv 0$ and $r>0$. Furthermore, select $\lambda_{1}:=\frac{a^{2}}{r}$, and $\lambda_{2}:=c^{2}$ for $c \neq 0$ or merely $\lambda_{2} \in(0,1)$ when $c=0$. If there exist $M>1$ and $\lambda>0 \quad$ satisfying $\tau\left(\lambda+r+\frac{\lambda_{1}}{\lambda_{2}} e^{\lambda \breve{d}}\right)<-\ln \lambda_{2}, \quad$ and $\tau\left(\lambda+r+\lambda_{1} M e^{-\lambda \tau}\right)<-\ln M$, then the dynamic system (10) is UGES such that $\|\xi(t)\| \leq \sqrt{M}\left\|\xi_{t_{0}}\right\| e^{-\frac{\lambda}{2}\left(t-t_{0}\right)}$ for all $t \geq t_{0}$.

Bringing together Lemma 1 and [30], yields:

Theorem 1: Assume that (10) is UGES with parameters $M>1$ and $\lambda>0$ along with $\sup _{t \in \mathbb{R}}\|\tilde{v}(t)\| \leq$ $\widetilde{K}_{v}$. Then the dynamics (10) is $\mathrm{L}_{p}$-stable with gain $\frac{2}{\lambda} \sqrt{M}$ and bias $\frac{\widetilde{\kappa}_{v} \sqrt{M}}{e^{\frac{\lambda \varepsilon}{2}}-1}$ from $\tilde{u}$ to $\xi$ for each $p \in[1, \infty]$.

\subsection{Applying the Small-Gain Theorem}

We now state and prove the main result of this article. After interconnecting $\Sigma_{n}$ and $\Sigma_{e}$ using $H\left(x_{t}, \omega_{t}\right)$ and $W(i, e)$ according to Fig. 2, we impose the small-gain condition so that the small-gain theorem can be invoked.

Theorem 2: Assume the UGES protocol, delays $d_{1}(t), \ldots d_{l}(t)$ and noise level $K_{v} \geq 0$ are given. Furthermore, suppose that

(a) there exists a continuous function

$H: P C\left([-\bar{d}, 0], \mathbb{R}^{n_{x}}\right) \times P C\left([-d, 0], \mathbb{R}^{n_{\omega}}\right) \rightarrow \mathbb{R}^{m}$

such that $\Sigma_{n}$ is $\mathrm{L}_{p}$-stable from $(W, \omega)$ to $H\left(x_{t}, \omega_{t}\right)$ for some $p \in[1, \infty]$, i.e., there exist $K_{H}, \gamma_{H} \geq 0$ such that $\left\|H\left[t_{0}, t\right]\right\|_{p} \leq K_{H}\left\|x_{t_{0}}\right\|+\gamma_{H}\left\|(W, \omega)\left[t_{0}, t\right]\right\|_{p}$,

for all $t \geq t_{0}$, and

(b) there exists $L \geq 0$ and $d: \mathbb{R} \rightarrow \mathbb{R}_{+}, \sup _{t \in \mathbb{R}} d(t)=\breve{d}$, such that for almost all $t \geq t_{0}$, almost alle $\in \mathbb{R}^{n_{e}}$ and for $\operatorname{all}\left(i, x_{t}, \omega_{t}\right) \in$

$\mathbb{N}_{0} \times P C\left([-\bar{d}, 0], \mathbb{R}^{n_{x}}\right) \times P C\left([-d, 0], \mathbb{R}^{n_{\omega}}\right)$ it holds that $\left\langle\frac{\partial W(i, e)}{\partial e}, g\left(t, x_{t}, e_{t}, \omega_{t}\right)\right\rangle \leq L W(i, e(t-d(t)))+\left\|H\left(x_{t}, \omega_{t}\right)\right\|$.

Then the NCS (5) is $\mathrm{L}_{p}$-stable from $\omega$ to $(H, e)$ with gain $\frac{\gamma_{H}\left(1+\frac{\gamma_{W}}{\underline{a}}\right)}{1-\gamma_{W} \gamma_{H}}$ and bias $\frac{\gamma_{H}+\frac{1}{a}}{1-\gamma_{W} \gamma_{H}} \frac{\bar{a} K_{v} \sqrt{M}}{e^{\frac{\lambda \varepsilon}{2}}-1}$ for each $\tau$ for which there exist $M>1$ and $\lambda>0$ satisfying (I), (II) and $\frac{2}{\lambda} \sqrt{M} \gamma_{H}<1$ with parameters $a=\frac{\bar{a}}{\underline{a}} L$ and $c=\rho$.

Remark 1: The condition (a) of Theorem 2 imposes a robustness requirement on the nominal system $\Sigma_{n}$ against the network-induced imperfections listed in Section 3 and disturbances considered herein. The condition (b) of Theorem 2, related to the error system $\Sigma_{e}$, establishes a connection between the current growth rate of $W(i, e)$ and its past values.

$$
f\left(t, x_{t}, e, \omega\right):=\left[\begin{array}{c}
f_{p}\left(t, x_{p_{t}}, g_{c_{t}}\left(t, x_{c_{t}}\right)+e_{u}(t), \omega_{p}(t)\right) \\
f_{c}\left(t, x_{c_{t}}, g_{p_{t}}\left(t, x_{p_{t}}\right)+e_{y}(t), \omega_{c}(t)\right)
\end{array}\right]=:\left[\begin{array}{l}
f_{1}\left(t, x_{t}, e, \omega\right) \\
f_{2}\left(t, x_{t}, e, \omega\right)
\end{array}\right] ; \quad h(t, e(t)):=\left[\begin{array}{l}
h_{y}(t, e(t)) \\
h_{u}(t, e(t))
\end{array}\right]
$$




$$
\begin{aligned}
& g\left(t, x_{t}, e_{t}, \omega_{t}\right):= \\
& :=\left[\begin{array}{l}
\hat{f}_{p}\left(t, x_{p_{t}}, x_{c_{t}}, g_{p_{t}}\left(t, x_{p_{t}}\right)+e_{y_{t}}, g_{c_{t}}\left(t, x_{c_{t}}\right)+e_{u_{t}}, \omega(t)\right)-\left(\frac{\partial g_{p}}{\partial t}\right)_{t}\left(t, x_{p_{t}}\right)-\left(\frac{\partial g_{p}}{\partial x_{p}}\right)_{t}\left(t, x_{p_{t}}\right) f_{1_{t}}\left(t, x_{t}, e, \omega\right) \\
\hat{f}_{c}\left(t, x_{p_{t}}, x_{c_{t}}, g_{p_{t}}\left(t, x_{p_{t}}\right)+e_{y_{t}}, g_{c_{t}}\left(t, x_{c_{t}}\right)+e_{u_{t}}, \omega(t)\right)-\left(\frac{\partial g_{c}}{\partial t}\right)_{t}\left(t, x_{c_{t}}\right)-\left(\frac{\partial g_{c}}{\partial x_{c}}\right)_{t}\left(t, x_{c_{t}}\right) f_{2_{t}}\left(t, x_{t}, e, \omega\right)
\end{array}\right]
\end{aligned}
$$

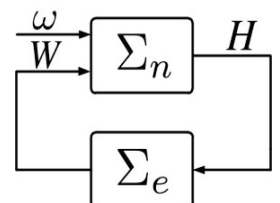

Figure 2 The nominal system $\Sigma_{n}$ interconnected with the error system $\Sigma_{\theta}$

Remark 2: The quantization process introduces quantization errors that can be readily integrated into bias provided these errors are bounded. As inferred in [31], feedback control with quantization errors cannot yield closed-loop robustness with linear $\mathrm{L}_{p}$-gains. In other words, the bias term in Theorem 2 is needed and cannot be removed without contradicting [31].

Remark 3: Lossy communication channels with an upper bound on the number of successive dropouts, say $N_{d} \in \mathbb{N}$, are readily included in $\tau$ from Theorem 2 . Asynchronous transmissions among NCS links are also readily included in $\tau$ from Theorem 2 (see [9] for more). Observe that asynchronous transmission protocols increase the likelihood of packet collisions [10].

At times, it is important to design NCSs such that a prespecified/desired robustness level is attained, which brings us to the following result:

Corollary 1: Assume the conditions of Theorem 2 are met and $x$ is $\mathrm{L}_{p}$-detectable from $(W, \omega, H)$ with gain $\gamma_{d}$. Then the NCS (5) is $\mathrm{L}_{p}$-stable with bias from $\omega$ to $x$ with the desired $\mathrm{L}_{p}$-gain $\gamma_{\mathrm{des}}=\frac{\gamma_{d}}{1-\gamma_{W} \gamma_{H}}$.

\section{CASE STUDY}

The inverted pendulum, depicted in Fig. 3, is a classical nonlinear control process $[29,35]$. Consider the inverted pendulum of mass $m=1$ given by

$\dot{x}_{p 1}=x_{p 2}+\omega_{1}$,

$\dot{x}_{p 2}=\frac{1}{L}\left(-g \cos \left(x_{p 1}\right)+u\right)+\omega_{2}$,

where $L=2$ and $g=9.8$ whilst $\omega_{1}$ and $\omega_{2}$ capture modeling uncertainties and/or external disturbances, controlled with

$u=-L \lambda x_{p 2}+g \cos \left(x_{p 1}\right)-K\left(x_{p 2}+\lambda x_{p 1}\right)$,

where $\lambda=1$ and $K=50$ are chosen controller parameters [32]. In the absence of network-induced imperfections, the above control law provably keeps the pendulum at rest, that is, $x_{p 2}=0$, in the upright position, that is, $x_{p 1}=$ 0 . However, can this goal be achieved in the network settings? If it can, which robustness levels are attained for the given controller parameters and a set of network phenomena (e.g., scheduling protocols, sampled, corrupted, delayed and lossy data, etc.)? Note that the knowledge of $\mathrm{L}_{p}$-gains allows one to quantify maximal deviations of the pendulum from the goal point $\left(x_{p 1}, x_{p 2}\right)=(0,0)$ given the $\mathrm{L}_{p}$-norm of disturbances $\omega(t):=\left(\omega_{1}(t), \omega_{2}(t)\right)$. In other words, not only we know that greater disturbances result in greater deviations, but we can also compute upper bounds on these deviations when bounded disturbances are present.

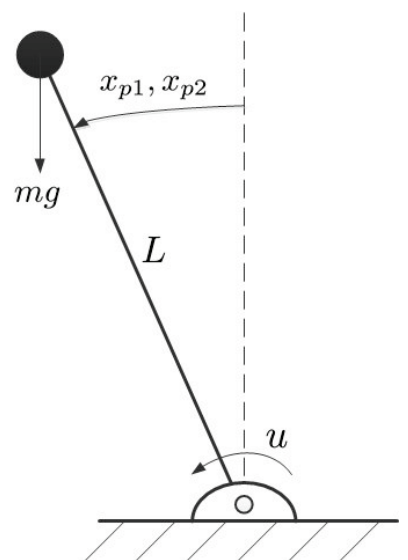

Figure 3 An illustration of the inverted pendulum.

Suppose that noisy values of $x_{p 1}$ and $x_{p 2}$ are transmitted over a communication network while the control signal is transmitted via a dedicated point-to-point connection without delays nor distortions, that is, $\hat{u}=u$. In addition, suppose that the measurements of $x_{p 2}$ arrive at the controller either with a constant delayd $\geq 0$ or with a time-varying delay $d(t) \leq \breve{d}$ such that $|\dot{d}(t)| \leq \breve{d}_{1}=$ 0.5. On the other hand, suppose the sampled measurements of $x_{p 1}$ arrive instantaneously. Apparently, there are two NCS links and we have $l=2$. Namely, one NCS link transmits information regarding $x_{p 1}$ whilst the other NCS link transmits information regarding $x_{p 2}$. Because this controller is without internal dynamics, we take $x(t):=x_{p}(t)=\left(x_{p 1}(t), x_{p 2}(t)\right)$. Lastly, we point out that $p=2$ is considered throughout this case study.

Owing to two NCS links, the corresponding error vector is (compare with (3))

$e=\left[\begin{array}{l}e_{1} \\ e_{2}\end{array}\right]=\hat{y}-(\underbrace{\left[\begin{array}{c}x_{p 1}(t) \\ 0\end{array}\right]+\left[\begin{array}{c}0 \\ x_{p 2}(t-d(t))\end{array}\right]}_{y_{t}})$,

while expressions (6) and (7) are

$\dot{x}(t)=\underbrace{\left[\begin{array}{cc}0 & 1 \\ \frac{-K \lambda}{L} & -1\end{array}\right]}_{A_{1}} x(t)+\underbrace{\left[\begin{array}{cc}0 & 0 \\ 0 & \frac{-K}{L}-\lambda L\end{array}\right]}_{A_{2}} x(t-d(t))+$ 


$$
\begin{aligned}
& \left\langle\frac{\partial W_{T O D}(i, e)}{\partial e}, \dot{e}\right\rangle \leq \\
& \underbrace{\left(1+\breve{d}_{1}\right)\left(\|B\|+\frac{g}{L}\right)}_{L_{T O D}} \underbrace{\|e(t-d(t))\|}_{W_{T O D}(t, e(t-d(t)))}+ \\
& \underbrace{\left(\left\|B_{1} x(t)+C_{1} \omega(t)+(1-\dot{d}(t))\left(B_{2} x(t-d(t))+B_{3} x(t-2 d(t))+C_{2} \omega(t-d(t))\right)\right\|\right)}_{H_{T O D}\left(x_{t}, \omega_{t}\right)}, \\
& \left\langle\frac{\partial W_{R R}(i, e)}{\partial e}, \dot{e}\right\rangle \leq \\
& \underbrace{\sqrt{l}\left(1+\breve{d}_{1}\right)\left(\|B\|+\frac{g}{L}\right)}_{L_{T O D}} \underbrace{\|D(i) e(t-d(t))\|}_{W_{R R}(t, e(t-d(t)))}+ \\
& \underbrace{\sqrt{l}\left(\left\|B_{1} x(t)+C_{1} \omega(t)+(1-\dot{d}(t))\left(B_{2} x(t-d(t))+B_{3} x(t-2 d(t))+C_{2} \omega(t-d(t))\right)\right\|\right)}_{H_{R R}\left(x_{t}, \omega_{t}\right)},
\end{aligned}
$$

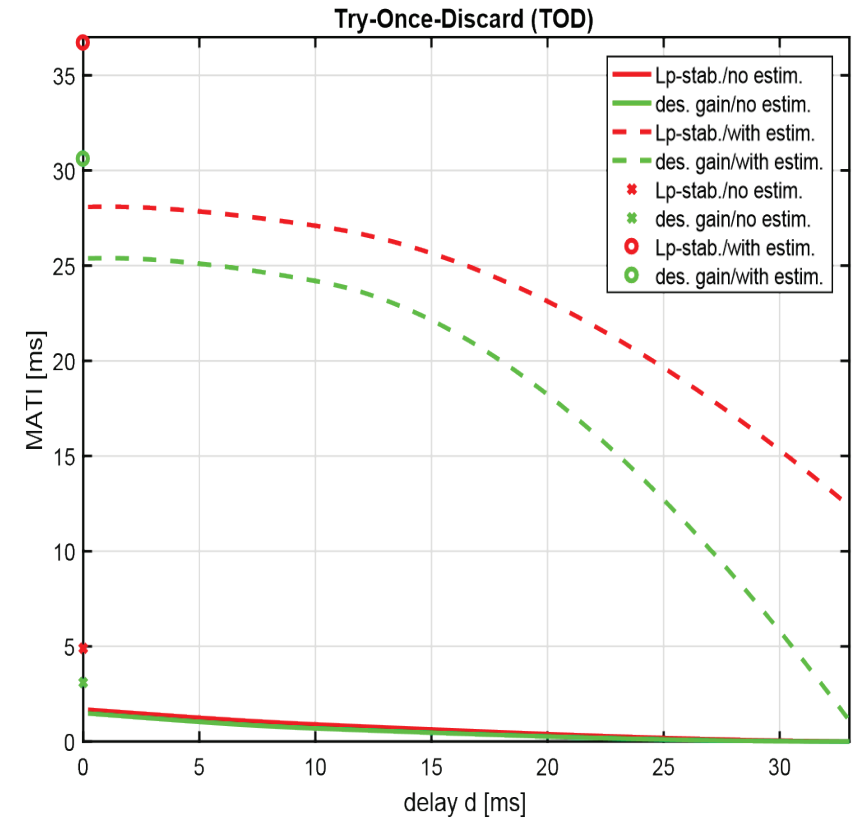

(a)

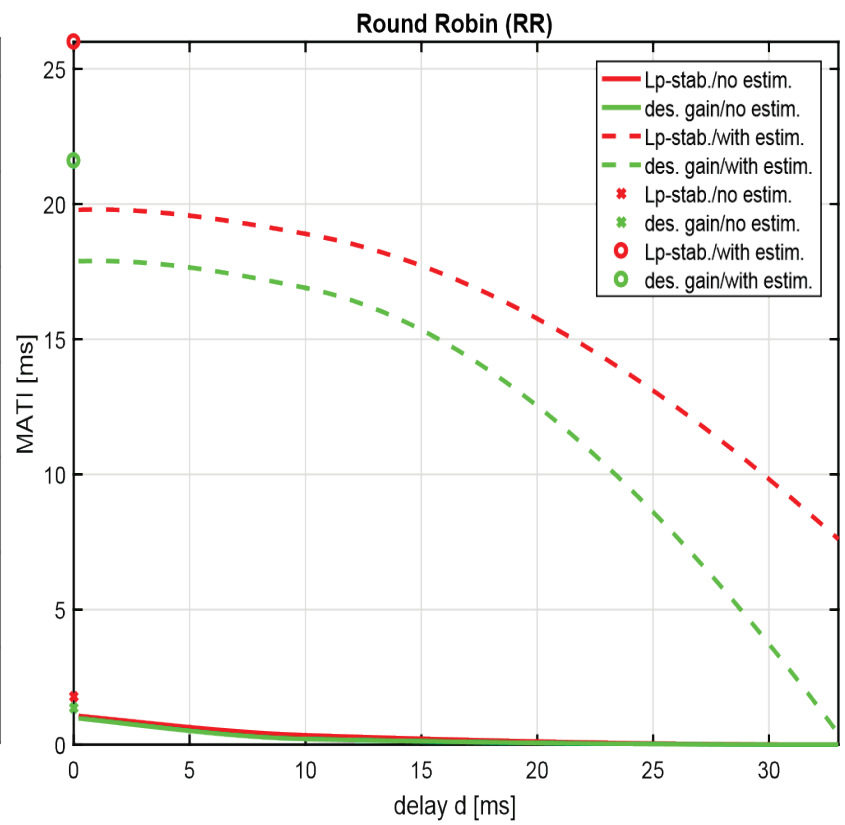

(b)

Figure 4 MATI-delay pairs with constant delays $d \geq 0$ yielding the same $L_{p}$-gains in scenarios with and without estimation: (a) TOD; and, (b) RR. The red lines correspond to robustness with an unspecified (i.e., arbitrary large but finite) desired $\mathrm{L}_{p}$-gain $\gamma_{\text {des }}$ while the green lines correspond to $\gamma_{\text {des }}=15$.

$$
+\left[\begin{array}{c}
0 \\
n\left(x_{1}(t), e_{1}(t)\right)
\end{array}\right]+\underbrace{\left[\begin{array}{cc}
0 & 0 \\
\frac{-K \lambda}{L} & \frac{-K}{L}-\lambda L
\end{array}\right]}_{B} e(t)+\omega(t),
$$$$
\dot{e}(t)=\dot{\hat{y}}+\underbrace{\left[\begin{array}{cc}
0 & -1 \\
0 & 0
\end{array}\right]}_{B_{1}} x(t)+\underbrace{\left[\begin{array}{cc}
-1 & 0 \\
0 & 0
\end{array}\right]}_{C_{1}} \omega(t)+
$$

$$
+(-B e(t-d(t))+\underbrace{\left[\begin{array}{cc}
0 & 0 \\
\frac{K \lambda}{L} & 0
\end{array}\right]}_{B_{2}} x(t-d(t))-
$$

$$
-\left[n\left(x_{1}(t-d(t)), e_{1}(t-d(t))\right)\right]+\underbrace{\left[\begin{array}{cc}
0 & 0 \\
0 & -1
\end{array}\right]}_{C_{2}} \omega(t-d(t))+
$$

$$
+\underbrace{\left[\begin{array}{cc}
0 & 0 \\
0 & \frac{K}{L}+\lambda L
\end{array}\right]}_{B_{3}} x(t-2 d(t)))(1-\dot{d}(t)),
$$

where

$$
n\left(x_{1}(t), e_{1}(t)\right)=\frac{-2 g}{L} \sin \left(\frac{e_{1}(t)+2 x_{1}(t)}{2}\right) \sin \left(\frac{e_{1}(t)}{2}\right) .
$$

According to [9], let us choose $W_{T O D}(t, e)$ $:=\|e\| \operatorname{and} W_{R R}(t, e):=\|D(i) e\|$, where $D(i)$ is a diagonal matrix with diagonal entries upper bounded by $\sqrt{l}$ and lower bounded by unity. Since $l=2$, the RR protocol alternates transmissions of $x_{p 1}$ and $x_{p 2}$ while the TOD protocol grants network access to the NCS link with greater noise-free uncorrupted error $\left\|e_{i}\right\|$. Clearly, TOD is more complex than RR. Thus, TOD may not be realizable in some real-life applications (e.g., when the scheduler cannot access unsullied information). Now, we compute $L_{T O D}, \quad H_{T O D}(e, \omega, d), \quad L_{R R}$ and $H_{T O D}(e, \omega, d)$ from Theorem 2 for the ZOH strategy and reach (12)-(11). The Lyapunov-Krasovskii functional utilized to estimate $\gamma_{H}$ is

$$
\begin{gathered}
V\left(t, x_{t}, \dot{x}_{t}\right)=x(t)^{T} P x(t)+\int_{t-\breve{d}}^{t} x(s)^{T} S x(s) \mathrm{d} s+ \\
+\breve{d} \int_{-\breve{d}}^{0} \int_{t+\theta}^{t} \dot{x}(s)^{T} R \dot{x}(s) \mathrm{d} s \mathrm{~d} \theta+\int_{t-d(t)}^{t} x(s)^{T} Q x(s) \mathrm{d} s,
\end{gathered}
$$

where $S, R$ and $Q$ are positive-semidefinite symmetric matrices while $P$ is a positive-definite symmetric matrix. The connection between Lyapunov-Krasovskii 
functionals and $\mathrm{L}_{2}$-gains is explained in, among others, [33, Chapter 6] and [34].

When employing model-based estimation, the following estimator is used (compare with (4))

$$
\begin{array}{r}
\dot{\hat{y}}=B \hat{y}(t-d(t))(1-\dot{d}(t))=B(e(t-d(t))+ \\
\left.+\left[\begin{array}{ll}
1 & 0 \\
0 & 0
\end{array}\right] x(t-d(t))+\left[\begin{array}{ll}
0 & 0 \\
0 & 1
\end{array}\right] x(t-2 d(t))\right)(1-\dot{d}(t)),
\end{array}
$$

which can be readily employed provided $d(t)$ is known. In order to obtain known delays, one can intentionally delay the communicated signals via time-stamping of data and introduction of buffers at receiver ends [2-4, 6].

After applying the methodology from Section 4, we obtain Figs.4 and 5 for scenarios with an unspecified $\gamma_{\text {des }}$ and with $\gamma_{\text {des }}=15$ specified a priori. Both figures provide evidence that the TOD protocol leads to greater robustness levels (at the expense of additional implementation costs and complexity [27]) and that model-based estimators significantly increase robustness margins in comparison with the $\mathrm{ZOH}$ strategy. In addition, since the scenarios with $d(t) \equiv 0$ boil down to
ODE modeling, we utilize less conservative tools for computing $\mathrm{L}_{2}$-gains obtaining greater $\mathrm{L}_{p}$-gains as visible in Fig. 4. The Maximally Allowable Transmission Intervals (MATIs), found on the $y$-axis of these plots, represent upper bounds of the stabilizing intervals between two consecutive data arrival times (see [7-9, 11, 18] for comprehensive discussions regarding MATIs). As expected, time-varying delays upper bounded with some $\breve{d}$ lead to increased control system sensitivity to external disturbances and noise when compared to constant delays $\breve{d}$. It is worth mentioning that $d(t) \equiv 33 \mathrm{~ms}$, for scenarios with constant delays, and $\bar{d} \equiv 33 \mathrm{~ms}$, for scenarios with time-varying delays, are the maximal values for which we are able to establish $\mathrm{L}_{p}$-stability, that is, robustness, with the selected Lyapunov-Krasovskii functional. However, a different Lyapunov-Krasovskii functional might lead to less conservative robustness margins. Altogether, in comparison with traditional control systems, more factors impact robustness levels of NCSs and all of them need to be accounted for during control system design and implementation.

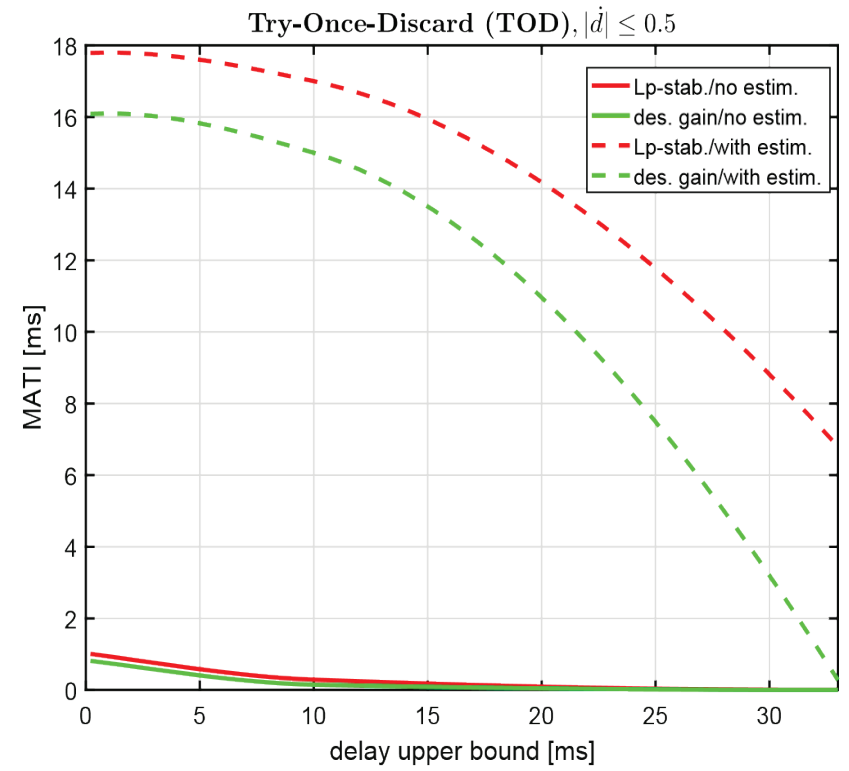

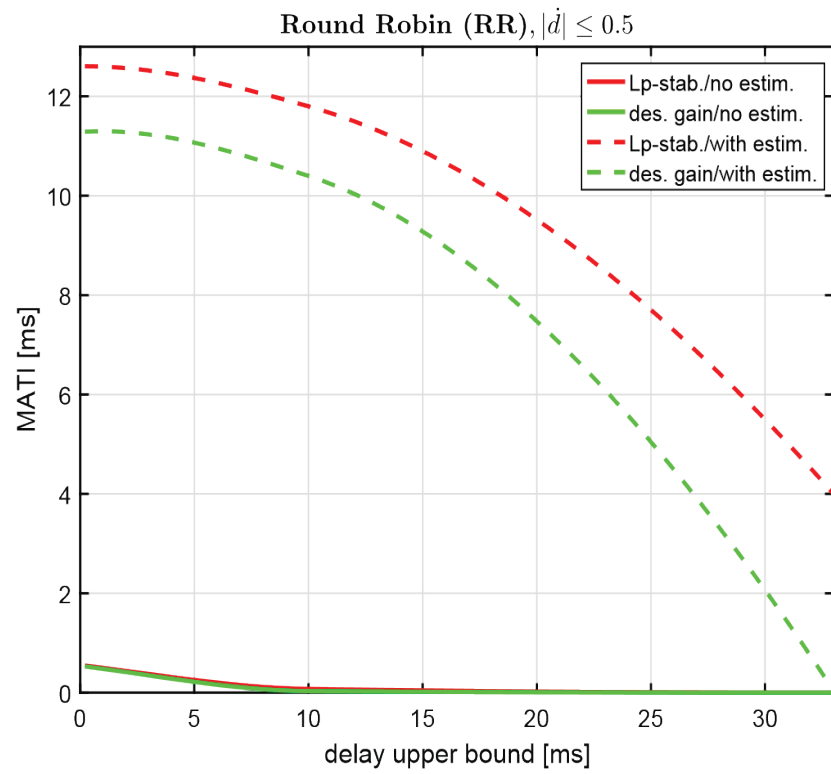

(b)

Figure 5 MATIs-delay pairs with time-varying delays $d(t)$ such that $d(t) \leq \breve{d}$ and $|\dot{d}(t)| \leq \breve{d}_{1}=0.5$ yielding the same $L_{p}$-gains in scenarios with and without estimation: (a) TOD; and, (b) RR. The red lines correspond to robustness with an unspecified (i.e., arbitrary large but finite) desired $L_{p}$-gain $\gamma_{\text {des }}$ while the green lines correspond to $\gamma_{\text {des }}=15$.

\section{CONCLUSION AND FUTURE WORK}

In this article, we extend our recent results and devise a procedure for measuring robustness of NCSs, in terms of $\mathrm{L}_{p}$-gains, with respect to the commonly encountered network-induced imperfections. Our procedure employs Lyapunov-based arguments followed by the small-gain theorem and is applicable to a large class of control systems and network phenomena. Namely, we consider nonlinear time-varying dynamic controllers and plants, UGES scheduling protocols, intermittent sampling, output feedback and model-based estimation and as well as nonuniform time-varying large delays, quantization, packet dropouts and noisy data. Our approach allows control practitioners to accomplish required robustness levels by trading off control laws, scheduling protocols or model-based estimators against network-induced imperfections. The inverted pendulum case study illustrates the important aspects of the proposed approach.

The present approach can be enhanced by devising conditions for dynamic scheduling protocols based on the noisy signal values at data acquiring instants on the scheduler end, rather than on noiseless signal values at data arrival instants on the NCS link ends. At the moment, the arbitration for dynamic protocols involves prediction of error resets of noise-free data at data arrival instants, which might not be implementable in many reallife applications. Also, Remark 2 merely scratches the surface of quantized feedback control and further investigations in this direction are needful. 


\section{ACKNOWLEDGEMENTS}

This research has been partly supported by the Ministry of Science, Education and Sports of the Republic of Croatia under the grant "Centre of Research Excellence for Data Science and Cooperative Systems".

\section{REFERENCES}

[1] Hirche, S., Matiakis, T., \& Buss, M. (2009). A distributed controller approach for delay-independent stability of networked control systems. Automatica, 45(5), pp. 18281836. https://doi.org/10.1016/j.automatica.2009.04.016

[2] Bemporad, A., Heemels, W. P. M. H., \& VejdemoJohansson, M. (2010). Networked Control Systems. Ser. Lecture Notes in Control and Information Sciences, vol. 406, Springer-Verlag, London. https://doi.org/10.1007/978-0-85729-033-5

[3] Xia, Y., Fu, M., \& Liu, G. (2011). Analysis and Synthesis of Networked Control Systems. Ser. Lecture Notes in Control and Information Sciences, 409, Springer-Verlag, Berlin Heidelberg. https://doi.org/10.1007/978-3-642-17925-9_8

[4] Longo, S., Su, T., Herrmann, G., \& Barber, P. (2013). Optimal and Robust Scheduling for Networked Control Systems. Ser. Automation and Ctrl. Engineering. Taylor \& Francis,

[5] Sauter, D., Sid, M., Aberkane, S., \& Maquin, D. (2013). Co-design of safe networked control systems. Annual Reviews in Control, 37(2), 321-332. https://doi.org/10.1016/j.arcontrol.2013.09.010

[6] Fridman, E. (2014). Introduction to Time-Delay Systems. Analysis and Control. Ser. Systems \& Control: Foundations $\&$ Applications. Springer International Publishing. https://doi.org/10.1007/978-3-319-09393-2

[7] Tolić, D., Sanfelice, R. G., \& Fierro, R. (2015). Inputoutput triggered control using Lp-stability over finite horizons. International Journal of Robust and Nonlinear Control, 25(14), 2299-2327. https://doi.org/10.1002/rnc.3203

[8] Tolić, D., Jeličić, V., \& Bilas, V. (2015). Resource management in cooperative multi-agent networks through self-triggering. IET Control Theory \& Applications, 9(6), 915-928. https://doi.org/10.1049/iet-cta.2014.0576

[9] Tolić, D. \& Hirche, S. (2017). Stabilizing transmission intervals for nonlinear delayed networked control systems. IEEE Transactions on Automatic Control, 62(1), 488-494. https://doi.org/10.1109/TAC.2016.2557077

[10] Mamduhi, M. H., Molin, A., Tolić, D., \& Hirche, S. (2017). Error-dependent data scheduling in resource-aware multiloop networked control systems. Automatica, 81, 209-216. https://doi.org/10.1016/j.automatica.2017.03.005

[11] Nešić, D. \& Teel, A. R. (2004). Input-output stability properties of Networked Control Systems. IEEE Transactions on Automatic Control, 49(10), 1650-1667. https://doi.org/10.1109/TAC.2004.835360

[12] Jentzen, A., Leber, F., Schneisgen, D., Berger, A., \& Siegmund, S. (2010). An improved maximum allowable transfer interval for Lp-stability of networked control systems. IEEE Trans. on Automatic Control, 55(1), 179184. https://doi.org/10.1109/TAC.2009.2033771

[13] Borgers, D. P. \& Heemels, W. P. M. H. (2014). Stability analysis of largescale networked control systems with local networks: A hybrid small gain approach. Hybrid Systems. Computation and Control (HSCC), 103-112.

[14] Yu, H. \& Antsaklis, P. J. (2013). Event-triggered output feedback control for networked control systems using passivity: Achieving $\mathrm{L}_{2}$ stability in the presence of communication delays and signal quantization. Automatica, 49(1), 30-38. https://doi.org/10.1016/j.automatica.2012.09.005

[15] Tabuada, P. (2007). Event-triggered real-time scheduling of stabilizing control tasks. IEEE Trans. on Automatic Control, 52(9), 1680-1685. https://doi.org/10.1109/TAC.2007.904277

[16] Anta, A. \& Tabuada, P. (2010). To sample or not to sample: Self-triggered control for nonlinear systems. IEEE Trans. on Automatic Control, 55(9), 2030-2042. https://doi.org/10.1109/TAC.2010.2042980

[17] Lemmon, M. (2010). Event-triggered Feedback in Control, Estimation, and Optimization. Ser. Lecture Notes in Control and Information Sciences. Eds. A. Bemporad, W. P. M. H. Heemels, and M. Johansson, 405, Springer Verlag. https://doi.org/10.1007/978-0-85729-033-5_9

[18] Heemels, W. P. M. H., Teel, A. R., de Wouw, N. V., \& Nešić, D. (2010). Networked Control Systems with communication constraints: Tradeoffs between transmission intervals, delays and performance. IEEE Transactions on Automatic Control, 55(8), 1781-1796. https://doi.org/10.1109/TAC.2010.2042352

[19] Mazenc, F., Malisoff, M., \& Dinh, T. N. (2013). Robustness of nonlinear systems with respect to delay and sampling of the controls. Automatica, 49(6), 1925-1931. https://doi.org/10.1016/j.automatica.2013.02.064

[20] Kruszewski, A., Jiang, W. J., Fridman, E., Richard, J. P., \& Toguyeni, A. (2012). A switched system approach to exponential stabilization through communication network. IEEE Trans. on Automatic Control, 20(4), 887-900. https://doi.org/10.1109/TCST.2011.2159793

[21] Zhang, B., Kruszewski, A., \& Richard, J. P. (2014). A novel control design for delayed teleoperation based on delay-scheduled Lyapunov-Krasovskii functionals. International Journal of Control, 87(8), 1694-1706. https://doi.org/10.1080/00207179.2014.883464

[22] Walsh, G., Ye, H., \& Bushnell, L. (2002). Stability analysis of Networked Control Systems. IEEE Transactions on Control Systems Technology, 10(3), 438-446. https://doi.org/10.1109/87.998034

[23] Hespanha, J., Naghshtabrizi, P., \& Yonggang, X. (2007). A survey of recent results in Networked Control Systems. Proceedings of the IEEE, 95(1), 138-162. https://doi.org/10.1109/JPROC.2006.887288

[24] Tolić, D. \& Palunko, I. (2016). Intermittent information in networked' control systems. International Conference on Smart Systems and Technologies (SST 2016) / Osijek, Croatia, 269-274. https://doi.org/10.1109/SST.2016.7765672

[25] Ballinger, G. H. (1999). Qualitative theory of impulsive delay differential equations. $\mathrm{PhD}$ dissertation, University of Waterloo, Canada.

[26] Jiang, Z. P., Teel, A. R., \& Praly, L. (1994). Small-gain theorem for ISS systems and applications. Mathematics of Control, Signals and Systems, 7(2), 95-120. https://doi.org/10.1007/BF01211469

[27] Christmann, D., Gotzhein, R., Siegmund, S., \& Wirth, F. (2014). Realization of Try-Once-Discard in wireless multihop networks. IEEE Transactions on Industrial Informatics, 10(1), 17-26. https://doi.org/10.1109/TII.2013.2281511

[28] Estrada, T. \& Antsaklis, P. J. (2010). Model-based control with intermittent feedback: Bridging the gap between continuous and instantaneous feedback. International Journal of Control, 83(12), 2588-2605. https://doi.org/10.1080/00207179.2010.535173

[29] Khalil, H. (2002). Nonlinear Systems, $3^{\text {rd }}$ ed. Prentice Hall.

[30] Anokhin, A., Berezansky, L., \& Braverman, E. (1995). Exponential stability of linear delay impulsive differential equations. Journal of Mathematical Analysis and Applications, 193(3), 923-941. https://doi.org/10.1006/jmaa.1995.1275 
[31] Martins, N. (2006). Finite gain $L_{p}$ stability requires analog control. Systems and Control Letters, 55(11), 949-954. https://doi.org/10.1016/j.sysconle.2006.06.002

[32] Tallapragada, P. \& Chopra, N. (2011). On event triggered trajectory tracking for control affine nonlinear systems. IEEE Conf. on Decision and Control / December 2011, 5377-5382. https://doi.org/10.1109/CDC.2011.6161531

[33] Boyd, S., El-Ghaoui, L., Feron, E., \& Balakrishnan, V. (1994). Linear matrix inequalities in systems and control theory. Philadelphia: SIAM. https://doi.org/10.1137/1.9781611970777

[34] Coutinho, D. F. \& de Souza, C. E. (2008). Delay-dependent robust stability and $\mathrm{L}_{2}$-gain analysis of a class of nonlinear time-delay systems. Automatica, 44(8), 2006-2018. https://doi.org/10.1016/j.automatica.2008.01.003

[35] Ata, B. \& Coban, R. (2015). Artificial Bee Colony Algorithm Based Linear Quadratic Optimal Controller Design for a Nonlinear Inverted Pendulum. International Journal of Intelligent Systems and Applications in Engineering, 3(1), 1-6. https://doi.org/10.18201/ijisae.87020

\section{Contact information:}

Domagoj TOLIĆ, PhD, Postdoctoral Researcher

Department of Electrical and Computer Engineering,

University of Dubrovnik

Ćira Carića 4, 20000 Dubrovnik, Croatia

domagoj.tolic@unidu.hr

Ivana PALUNKO, PhD, Assistant Professor

Department of Electrical and Computer Engineering,

University of Dubrovnik

Ćira Carića 4, 20000 Dubrovnik, Croatia

ivana.palunko@unidu.hr 